
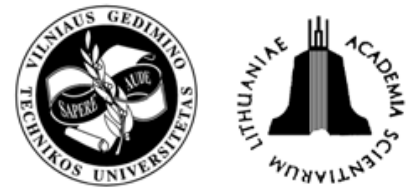

\title{
THE RESEARCH INTO HEAD INJURY CRITERIA DEPENDENCE ON CAR SPEED
}

\author{
Lina Pelenyte்-Vyšniauskienè $\dot{1}^{1}$, Algirdas Jurkauskas ${ }^{2}$ \\ Kaunas University of Technology, Panevėžys Institute, Klaipėdos g. 1, LT-35209 Panevėžys, Lithuania \\ E-mails: ${ }^{1}$ linapelenyte@gmail.com; ${ }^{2}$ algirdas.jurkauskas@ktu.lt
}

Received 20 May 2007; accepted 10 September 2007

\begin{abstract}
There are many ways of car collisions which depend on car motion modes before and after crashes, speed, kinds of baskets, their heights, weights and rigidity. The machinery of the occupant's movement at the moment of the crash is even more difficult. In order to find out precisely the chance of body injury, it is important to measure not only parameters that were mentioned above but also occupant's height, weight, age, position of sitting, condition of body, whether there was any protection system used.

The largest number of car crashes happen at the moment of Frontal Crash. This article's aim is to analyse the types of Frontal Crashes and their repartition, to diagnose what part in occupant's safety the protection system's use takes, and also to analyse head injury coefficient dependence on car speed and show critical injuries and fatality limits in cases when driver is driving with no seat-belts in and while the car is without airbag. The research is done at the moment of ideal Frontal Crash by simulating distance from the occupant body to the wheel in different types of baskets.
\end{abstract}

Keywords: frontal crash, occupants injuries, HIC, AIS, speed.

\section{Introduction}

In 1893 the first traffic accident was registered in the USA, the second one in 1896 in Europe. At the moment of last-mentioned accident car was going at $6,4 \mathrm{~km} / \mathrm{h}$.

It was more than 100 years ago when accidents were counted in such small numbers, nowadays this number reaches millions. In EU traffic includes more than 450 million drivers. Over the year about 1,3 million traffic accidents happen in which more than 0,4 million occupants are being killed and 1,7 million injured.

Car manufacturers are paying more and more attention to occupant's safety and organizations like NHTSA (National Highway Traffic Safety Administration), NCAP (New Car Assesment Program), Euro NCAP (European New Car Assessment Programme), etc. are testing cars, so that market gets only that car which passes the safety regulations. Certainly, this is not enough to se- cure occupant's health and life because the number of cars and their speed scope are increasing.

\section{Analysis of Frontal Crash}

The largest number of accidents happen at the moment of Frontal Crash (about $60 \%$ ).

In Table 1 most often Frontal Crash cases can be seen. In the first scheme (left) you can see car crashes into fixed Frontal Barrier. It takes about $21 \%$ of all Frontal Crashes. The second and third schemes simulate Left and Right Offsets, when crash point varies from 0 to 30 degrees It takes about $34 \%$ of Left Offset and $35 \%$ of Right Offset of all Frontal Crashes. In the fourth scheme you can see Left/Right crash, when the angle of crash trajectory is more than $30 \%$. It takes about $9 \%$ of all Frontal Crashes [1].

Table 1. Possible Frontal Crash distribution

Frontal Barrier, \pm 30 Degrees




\section{Injury tolerance limits}

During car crashes injuries are usually dangerous. Up to $10-12 \%$ of injured occupants die during crash because of heavy injuries.

Injury tolerance limits describe items such as fractures, injuries of organs, and other injuries. A classification is done via the Abbreviated Injury Scale $(A I S)$ or Overall Abbreviated Injury Scale (OAIS). With the AIS or OAIS, the single or total injury is described. The data span within a range from 0 to 6 . Table 2 shows the severity rating versus AIS.

Table 2. The abbreviated injury score

\begin{tabular}{|c|c|c|}
\hline AIS Code & Injury Level & Fatality Range \\
\hline 0 & no injury & $0.0 \%$ \\
\hline 1 & minor & $0.0-0.1 \%$ \\
\hline 2 & moderate & $0.1-0.4 \%$ \\
\hline 3 & serious & $0.8-2.1 \%$ \\
\hline 4 & severe & $7.9-10.6 \%$ \\
\hline 5 & critical & $53.1-58.4 \%$ \\
\hline 6 & maximum & virtually unsurvivable \\
\hline
\end{tabular}

The limits to the injury level depend on sex, anthropometrics, age, mass, mass distribution, and specific conditions [2].

The risk of head, thorax and abdominal injuries is significantly reduced in airbag-equipped cars compared to non-airbag cars. However, there is no concomitant reduction in the risk of upper and lower extremity injuries in airbag-equipped cars. In fact, the risk of lower extremity injuries (both KTH and below knee injuries) is higher among belted occupants in airbag - equipped cars than those with no airbag. This observation is similar to that found by Rupp et al. (2002). The risk of AIS 2+ injuries is highest to the lower extremities compared to any other body region in airbag-equipped cars. The risk of injury to the KTH (Knee - Thigh - Hip) complex is approximately the same as the risk of below knee injuries [3].

The risk of AIS 2+ injury to different body parts for different restraint environments is presented in Fig. 1. The risk of AIS 2+ injuries is higher to the unrestrained occupant than for the belted occupant in both airbag and no airbag-equipped cars.

According to NHTSA only the use of seat-belt reduces fatality risk up to $45 \%$ and airbag use reduces the risk up to additional $9 \%$. [4]. If driver's fatality risk without any protection system reaches $100 \%$, the risk of drivers using seat belt and airbag can be calculated using the formula:

$$
100 \cdot(1-0.45) \cdot(1-0.09)=50 \% .
$$

On the basis of NHSTA the given figures describe how and which particular systems reduce general fatality number in all kinds of Frontal Crashes (Table 3).

Having analyzed NHTSAs database of simulated crashes (465 cases), we managed to find out how injuries after the Frontal Crash spread (Fig. 2). It is obvious that at the moment of crash head and chest get the most severe damage [5].

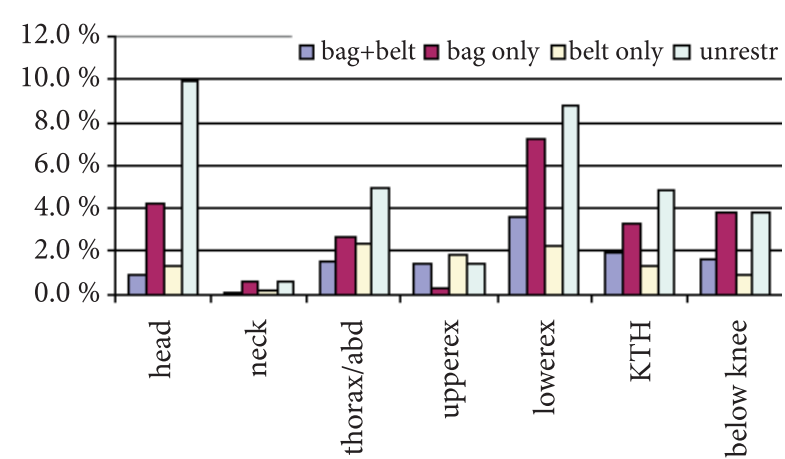

Fig. 1. Risk of AIS 2+ injuries in different body regions for different restraint environments in frontal crashes

Table 3. Death dependence on protection systems

\begin{tabular}{|c|c|}
\hline Protection system & Reduction of fatal accidents \\
\hline Airbag and seat-belt & $50 \%$ \\
\hline Only airbag & $14 \%$ \\
\hline Seat-belt & $45 \%$ \\
\hline
\end{tabular}

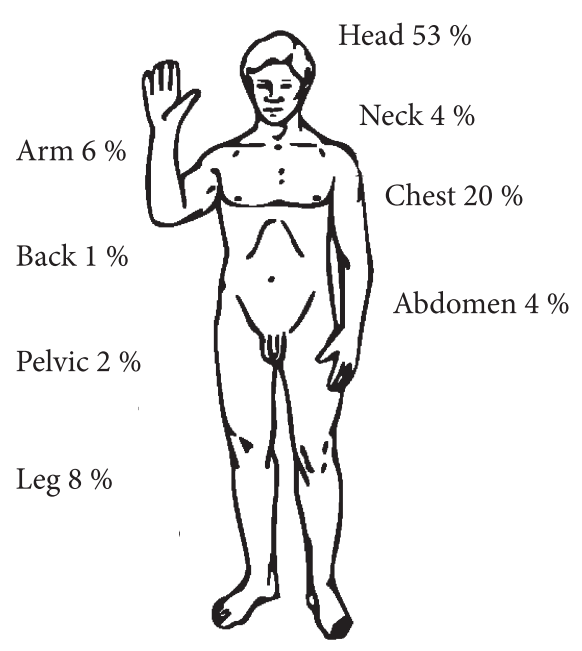

Fig. 2. Body region of unbelted occupants $(A I S=3)$

\section{The research on head injuries}

As we already detected, the head more often gets trauma. Head movements in the car are not limited by any restriction systems. Therefore, at the moment of crash it hits various objects in the car (window, wheel, dashboard, etc.). The hit of the head can cause bruises, scrapes, wounds, contusion and commotion of cerebrum, cranium cracks, cerebral haemorrhage, etc.

Encephalon injuries during motor transport accidents are mostly fatal for occupants up to 45 years old. When whole body injuries index $A I S=5$, they reach even $84 \%$ of whole body injuries [5].

According to the information of sixty researches there are many various criteria of injuries. Researches were lead by well-known scientists like Lissner, Lebow and Evans (1960), Ommaya and Hirsch (1971) and others.

Classic HIC (Head Injury Criteria) were given in addition to Vienna institute's index, specified model of the brain and medium tension criteria. On recommend- 
ed SAE (Safety of Automotive Engineers) basis head injury criteria were counted at every $3 \mathrm{~ms}$ time interval. The criterion was specified by Versace in 1971 and described by equation [6]:

$$
H I C=\left[\frac{1}{t_{2}-t_{1}} \cdot \int_{t_{1}}^{t_{2}} a d t\right]^{2.5} \cdot\left(t_{2}-t_{1}\right)
$$

where: $t_{1}$ and $t_{2}$ - any time moments during impact between the earlier mentioned $t_{\text {start }}$ and end $t_{\text {end }}$ times; $a-$ acceleration in time interval $t_{1}-t_{2}$.

In Table 4 you can see the values of HIC for different sex and age occupants:

$\mathrm{HIC}_{36}$ - the head is injured over $36 \mathrm{~ms}$;

$H I C_{15}$ - the head is injured over $15 \mathrm{~ms}$.

Table 4. $H I C$ values, when $A I S=5-6$

\begin{tabular}{|c|c|c|c|c|c|}
\hline$H I C$ & Male & Female & 6 year & 3 year & 12 Month \\
\hline$H I C_{36}$ & 1000 & 1000 & 1000 & 1000 & 660 \\
\hline$H I C_{15}$ & 700 & 700 & 700 & 570 & 390 \\
\hline
\end{tabular}

\section{HIC dependence on speed in various kinds of cars}

The essence of this research is to find out occupant's minimum possible deceleration constants of the acceleration, using common occupant's body forward movement [7]. It is known that occupant's deceleration of acceleration can be found out not only by taking measures or simulating the situation but using diagrams as well.

Calculating occupant's body movement at the moment of Frontal Crash, you need to pay attention to sequence of occupant's body movement:

The car hits the obstruction. The bumper gets deformed while the power of hit exceeding several times the car weight, stops it.

Occupant starts moving in the same direction as car was going. His speed is equal to car speed before the hit.

The car starts the backward movement, but occupant from inertia is still moving forward, when finally hits the parts of interior. Since the car moves backward after the hit, occupant's forward movement becomes shorter.

Parts of interior absorb part of occupant's kinetic energy and body starts moving backwards. Depending on the beginning of occupant's deceleration of acceleration and its growth, a part of car's general deformation length is being used.

Simplified occupant's body model centres of three masses are used for mathematical analysis. Supposing, that driver's height and weight are of average mean and he is not using any protection systems. Supposing, that head is going to hit the wheel in the direction B (Fig. 3) against no less than $25 \%$ of its square.

The ideal Frontal Crash is being analysed, when car cabin remains undamaged and all-in-one occupant's body is moving in rectilinear movement. Suppose, that occupant's movement acceleration until the hit is constant.
Occupant having a certain mass is displaced (covers a certain distance):

$$
s=s_{\text {interior total }}+s_{F z\left(t_{\text {thorax rebound }}\right)}-s_{\text {thorax }\left(t_{\text {start }}\right)},
$$

where:

$s_{\text {interior total }}$ - obstacle, distance in a car from thorax (body) until the closest obstacle, where occupant can be displaced;

$s_{F z\left(t_{\text {thorax }} \text { rebound }\right)}$ - distance, that the car covers, until the collision moment when thorax hits an obstacle;

$s_{\text {thorax }\left(t_{\text {start }}\right)}$ - distance that thorax (body) covers at the initial moment of collision.

Time, required to reach the final point and kinetic energy disappearance is equal [8] to:

$$
t_{\text {end }}=t_{\text {start }}+\left(\frac{2 \cdot s_{\text {req }}}{a_{\text {const }}}\right)^{0.5}
$$

$s_{\text {req }}$ - distance, required for the body not to be injured during the accident. It is calculated by the following formula:

$$
s_{r e q}=\frac{1}{2} v \cdot t=\frac{1}{2} a \cdot t^{2}=\frac{E_{k i n}}{m \cdot a_{\text {const }}} .
$$

Time at the beginning of collision $t_{\text {start }}$, during which thorax (occupant) is displaced by the distance $s_{\text {thorax }\left(t_{\text {start }}\right)}$, acceleration function:

$$
t_{\text {start }}=f\left(a_{\text {thorax }}=a_{\text {const }}\right) .
$$

Motion acceleration can be expressed by ratio $a=\frac{a_{t}}{g}$, then in a general case occupant's motion during the accident is $s=s_{0}+v_{0} \cdot t+\frac{1}{2} a \cdot t^{2}$, but as analysis shows, it is simplified $(a=$ const $)$, so $s=\frac{1}{2} a \cdot t^{2}$. Occupant's motion is $v=v_{0}+a \cdot t$. Having in mind $a=$ const, so $v=a \cdot t$. Occupant's motion travel during the accident is $s=\frac{v^{2}}{2 a}$.

However an occupant during the accident moves in the car interior, so his maximum travel is as long as up to the closest obstacle (windshield, panel, etc.). Time required to gain the initial acceleration equals the ratio of velocity and acceleration $t=\frac{v}{a}$. Since occupant is likely to move up to an obstacle, opposing system will start functioning when occupant moves in the distance $s$ until the obstacle. According to formula (2) $t_{2}=t_{1}+\sqrt[2]{\frac{2 s}{a}}$, as $t_{1}=0$ so $t_{2}=\sqrt[2]{\frac{2 s}{a}}$. Occupant's velocity on impact is $v=$
$a \cdot t_{2}$. 


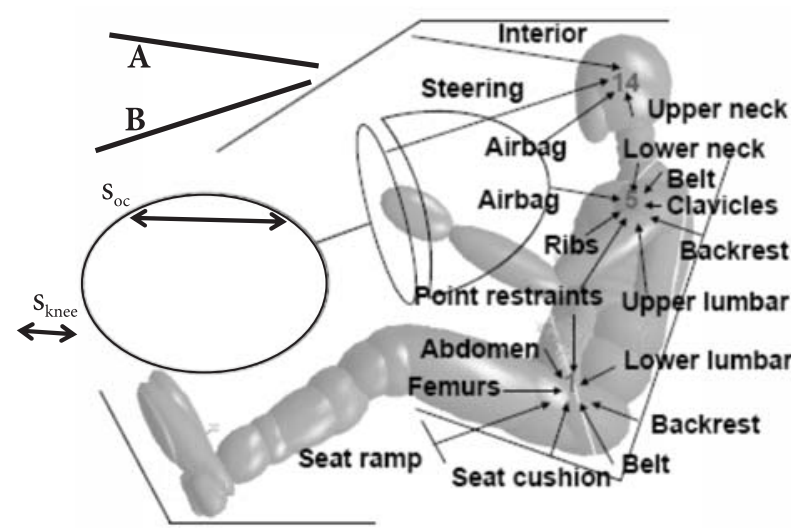

Fig. 3. Occupant's position in the car

Table 5. Measures of examined cars

\begin{tabular}{|l|c|c|c|c|}
\hline \multicolumn{1}{|c|}{ Model } & Year & Weight, $\mathrm{kg}$ & $s_{\max }$ & $s_{\min }$ \\
\hline Peugeot 605 & 1991 & 1460 & 65 & 40 \\
\hline VW Golf & 1995 & 1120 & 55 & 34 \\
\hline Citroen C25 & 1990 & 2170 & 43 & 27 \\
\hline Mazda 323 F & 1990 & 990 & 53 & 33 \\
\hline Lancia Zeta & 1997 & 690 & 43 & 22 \\
\hline BMW 524 & 1990 & 1500 & 48 & 26 \\
\hline Opel Astra & 1991 & 950 & 50 & 36 \\
\hline Audi A6 & 1996 & 1970 & 48 & 30 \\
\hline
\end{tabular}

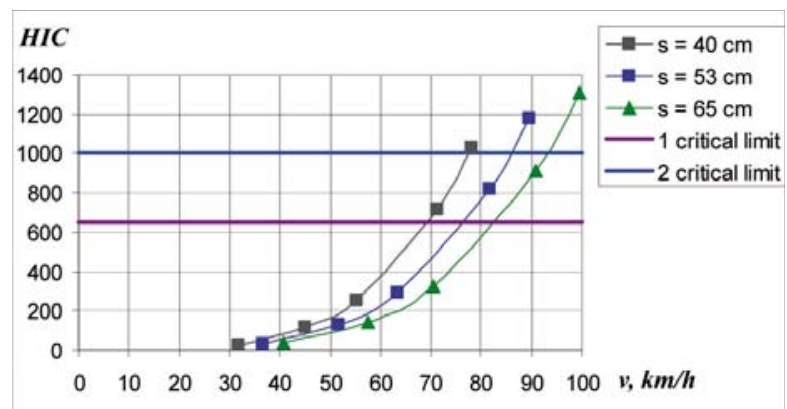

Fig. 4. HIC dependability on velocity (Peugeot 605)

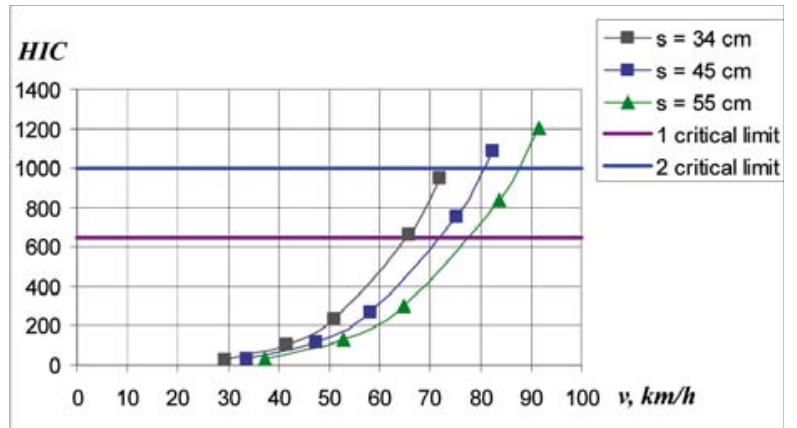

Fig. 5. HIC dependability on velocity (VW Golf)

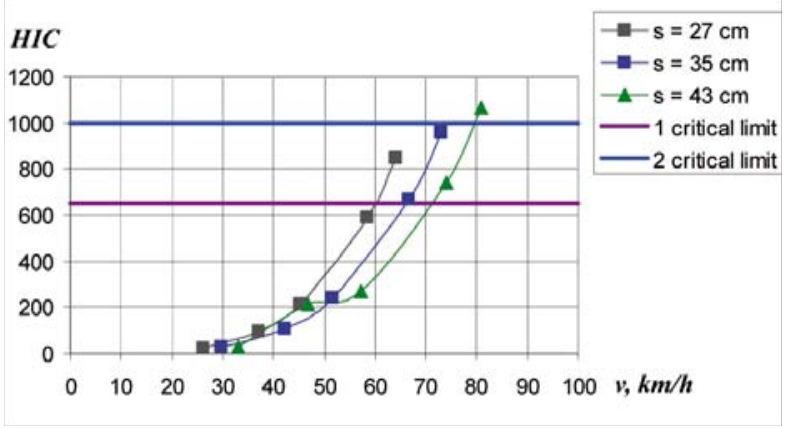

Fig. 6. HIC dependability on velocity (Citroen C25)

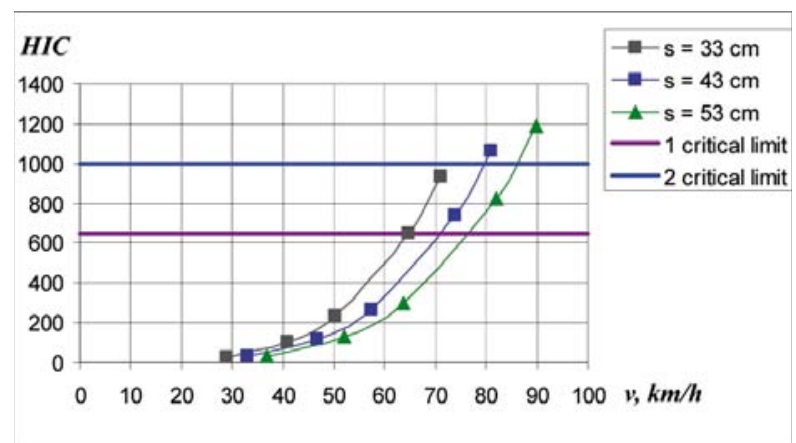

Fig. 7. HIC dependability on velocity (Mazda $323 \mathrm{~F}$ )

Using the formulas it is possible to calculate occupant's motion accelerations, their duration, occupant's motion during impact against opposing system and HIC. Since the acceleration is invariable so its volumes are selected.

Calculation in a few cases is given as $a_{1}=10 \mathrm{~g}, 20 \mathrm{~g}$, $30 \mathrm{~g}, 40 \mathrm{~g}, 50 \mathrm{~g}, 60 \mathrm{~g}$.

For the purpose of research 8 measures of different makes of cars and sizes were taken (Table 5). Measured distances from the outward man chest to the steering wheel are $s_{\max }$ and $s_{\min }$ (Fig. 3 and Table 5).

According to the given procedure we will count all measured cars $a\left(\mathrm{~m} / \mathrm{s}^{2}\right), t_{2},(\mathrm{~s}), v,(\mathrm{~m} / \mathrm{s})$ and $H I C$, when distance $s$ between driver and steering wheel is minimal, medium and maximal.

The calculated head injuries coefficient HIC and its dependence on speed, are shown in Figs. 4-11. This is speed when body will get certain value of injury. In given figures two critical values of injuries can be seen: the first critical limit shows that when $H I C<650$, head injuries are not fatal, brains are not damaged. The second limit shows that when $H I C \geq 1000$, head injuries are possibly heavy: cracked cranium, bone breaks, heavy concussion and cerebral haemorrhage can be observed. One of six victims of the accident dies at the moment of crash [9].

According to given figures, we can see that if the occupant until the resistance of protection systems goes undisturbed up to $60-80 \mathrm{~km} / \mathrm{h}$, in this case we get larger HIC values while $s$ value is lower. Yet when speed is higher, the chance of injury also gets higher when we have $s_{\max }$.

If after the crash, which matches the analysed ideal Frontal Crash case, there is a possibility to find out how 


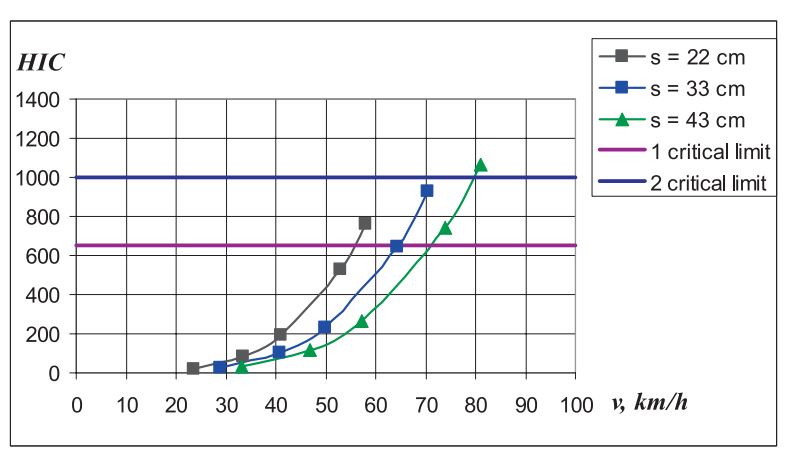

Fig. 8. HIC dependability on velocity (Lancia Zeta)

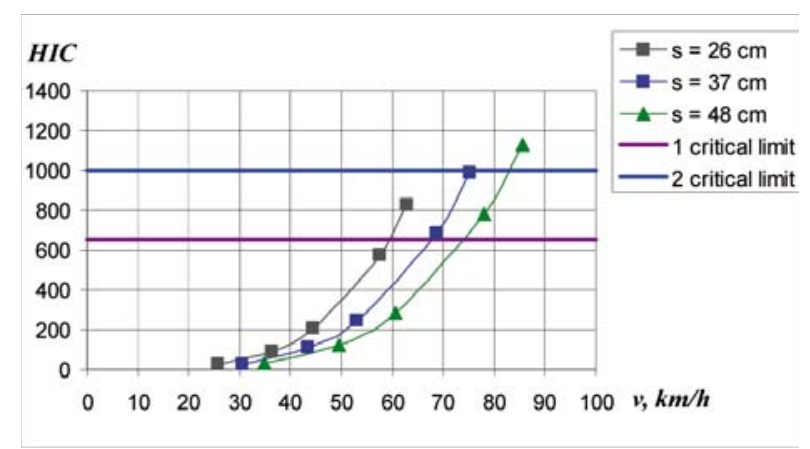

Fig. 9. HIC dependability on velocity (BMW 524)

far away the driver was sitting, so that having in mind his injuries value we can find out approximate car speed before the crash.

In Fig. 12 you can see average distances between the occupant and the wheel in the examined cars. It is clear that $s_{\max }$ is the highest in Peugeot 605 and the lowest in Lancia Zeta.

When occupant's speed before hitting the wheel is over $80 \mathrm{~km} / \mathrm{h}$ and the distance is medium, the highest HIC is observed in Peugeot: HIC $=1178$ and VW Golf: $H I C=1084$ (Fig. 13). The lowest possibility of injuries would be observed in Lancia Zeta: $H I C=927$.

\section{Conclusions}

1. According to the examination $60 \%$ of all crashes are frontal.

2. The use of airbag and seat-belt can decrease the possibility of death by $50 \%$.

3. Having analysed NHTSA database, such conclusions can be made: at the moment of crash, when AIS = $5-6$, the head is injured in $53 \%$ of cases and the chest is injured in $20 \%$ of cases.

4. After measuring 8 cabins of cars, the biggest and the smallest distance between occupant and wheel was found out.

5. After calculating every car's HIC dependence on speed, when $s=s_{\min }, s_{\text {vid }}$ and $s_{\max }$, we found out that the largest HIC is observed in Peugeot 605 and the lowest in Lancia Zeta. It is found that only two cars (from 8 cars) do not reach the second critical fatal zone.

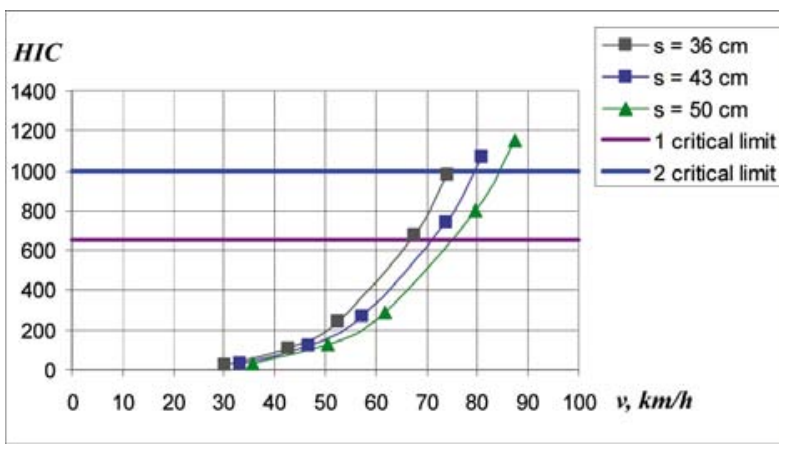

Fig. 10. HIC dependability on velocity (Opel Astra)

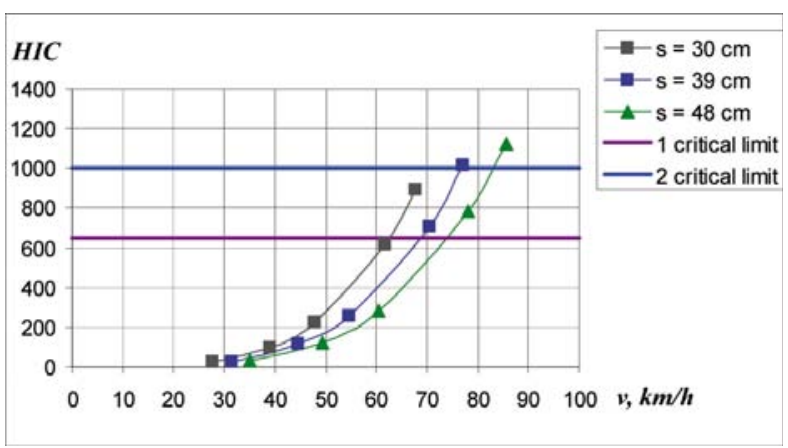

Fig. 11. HIC dependability on velocity (Audi A6)

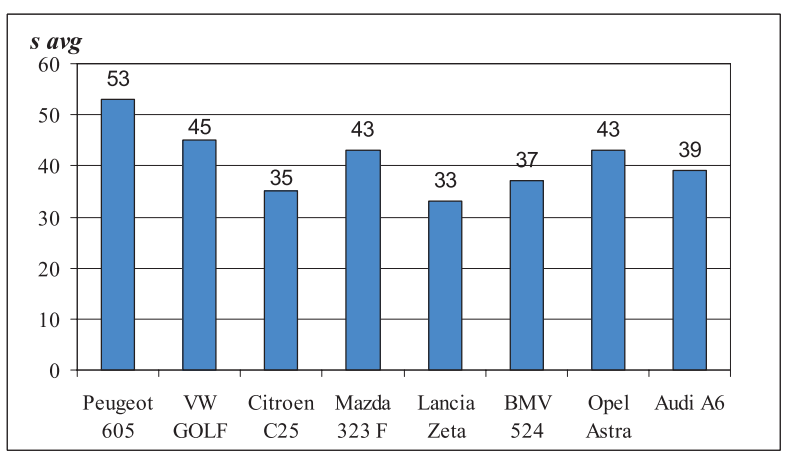

Fig. 12. Distribution of average distance $s$

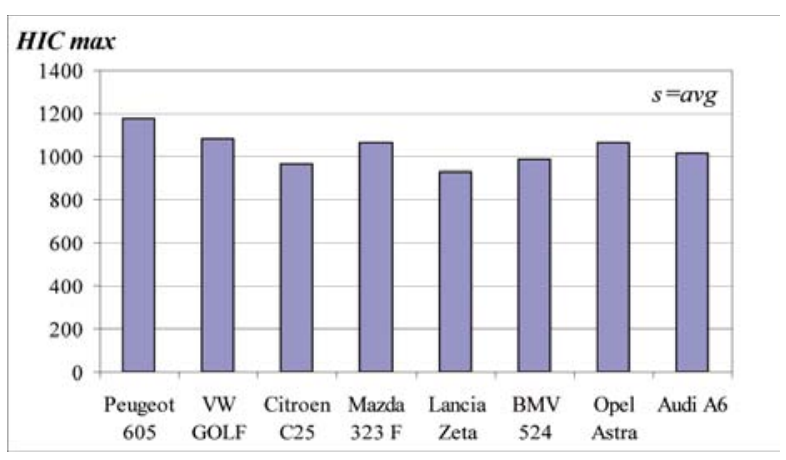

Fig. 13. Distribution of average distance $s$ in the cars 


\section{Resources}

1. SHELDON, L.; STUCKI, W.; HOLLOWELL, T. Determination of frontal offset test conditions based on crash data. National Highway Traffic Safety Administration, Information Systems and Services. Paper No. 98-S l-0-02. 1998, p. 15-35.

2. Association for the Advancement of Automotive Medicine. The Abbreviated Injury Scale. Illinois: Revision Des Plaines, $1990.80 \mathrm{p}$.

3. SHASHI, K. An overview of knee - thigh - hip injuries in frontal crashes in the United States. National Highway Traffic Safety Administration, 2005. 416 p.

4. VIANO, D. C.; CULVER, C. C.; HAUT, R. C.; MELVIN, J. W.; BENDER, M.; CULVER, R. H.; LEVINE, R. S. Bolster impacts to the knee and tibia of occupant cadavers and an anthropomorphic dummy. In Proceedings of the 22nd Stapp Car Crash Conference, SAE Technical Paper No. 7780896. 1994, p. 403-428.

5. DANIEL, A. G. Analysis of NASS cases involving SUV rollovers with belted and unbelted occupants. Report of National Crash Analysis Center (FHWA/NHTSA). May 2002, p. 1-15.

6. OMMAYA, A. K.; HIRSCH, A. E. Tolerances for cerebral concussion from head impact and whiplash in primates. Journal of Biomechanics, 1971, Vol. 4, p. 13-20.

7. KAI, I. Innovative Energiemanagement Methoden zur Analyse und Verbesserung von Insassenschutzsystemen. Berlin: ISS - Fahzeugtechnik. 2000.

8. PELENYTĖ-VYŠNIAUSKIENĖ, L.; JURKAUSKAS, A. Research into occupant's motion in cars during crashes. Transport, 2004, Vol. XIX, No. 4, p. 184-190.

9. KOSHIRO, O.; MUNEKAZU, K. Influences of the physical parameters on the risk to injuries at low impact speed. Accident Analysis \& Prevention, July 2004, Vol. 28, Issue 4, p. 265-271. 